\title{
O desafio do aprimoramento da avaliação em aprendizagem ativa na formação em saúde
}

\author{
Monike Alves Lemes ${ }^{1}$, Elza de Fátima Ribeiro Higa², Maria José \\ Sanches Marin'2, Carlos Alberto Lazarini ${ }^{2}$, Joyce Fernanda Soares \\ Albino Ghezzi ${ }^{3}$ e Rodolfo de Oliveira Medeiros ${ }^{3}$ \\ ${ }^{1}$ Estudante de Medicina. Universidade de Marília, Brasil | monikealvesx3@gmail.com | \\ https://orcid.org/0000-0002-8769-0993 \\ ${ }^{2}$ Departamento de Enfermagem. Faculdade de Medicina de Marília, Brasil | \\ hirifael@gmail.com; marnadia@terra.com.br; carlos.lazarini@gmail.com | \\ https://orcid.org/0000-0001-5772-9597; https://orcid.org/0000-0001-6210-6941; \\ https://orcid.org/0000-0003-3010-4436 \\ 3 Pós-graduandos. Universidade Estadual Paulista Júlio de Mesquita Filho, Brasil \\ jo.albino1988@gmail.com; rodorfo.famema@hotmail.com | https://orcid.org/0000-0002- \\ 5808-613X; https://orcid.org/0000-0002-4930-684X
}

\begin{abstract}
Resumo: Introdução: é esperado que, na aprendizagem ativa, a avaliação seja uma ferramenta modulatória do processo de desenvolvimento do estudante, mas, comumente, essa é uma prática angustiante para docentes e estudantes. Objetivo: identificar sugestões de aprimoramento da prática avaliativa em aprendizagem ativa na graduação em saúde; Métodos: estudo qualitativo, realizado por meio de entrevistas individuais semiestruturadas, com 25 docentes dos cursos de Enfermagem e Medicina, em uma Instituição de Ensino Superior pública, da região centro oeste do interior paulista. Os dados foram interpretados pela Técnica de Análise de Conteúdo, na modalidade temática, de acordo com as etapas Préanálise (organização dos dados coletados por meio de sucessivas leituras), Exploração do material (identificação das categorias) e Tratamento dos resultados: inferência e interpretação (esclarecimento dos significados contidos nos dados coletados e articulação entre interpretação dos dados, referencial teórico e objetivos do estudo). Resultados: foram identificadas três categorias de aprimoramento da avaliação na aprendizagem ativa em saúde, sendo: Desenvolvimento docente (formação pedagógica docente, especialmente em avaliação afetiva e melhor preparo para lidar com os aspectos relacionais, afetivos, comunicacionais e de grupo), Adequação dos instrumentos e práticas avaliativas (padronização e implementação de instrumentos avaliativos e adesão por critérios de correção mais objetivos) e Orientação aos estudantes (meios de capacitar os estudantes a respeito das práticas avaliativas e seus objetivos, com destaque para autoavaliação e avaliação dos pares). Considerações finais: nota-se que 0 aprimoramento da prática avaliativa na aprendizagem ativa é indissociável da capacitação de docentes e estudantes e da adoção de práticas e instrumentos de avaliação padronizados e conhecidos por todos os seus atores.
\end{abstract}

Palavras-chave: Aprendizagem; Aprendizagem Baseada em Problemas; Avaliação Educacional.

\section{The Challenge of Improving Evaluation in Active Learning in Health Education}

Abstract: Introduction: It is expected that, in active learning, assessment will be a modulatory tool in the student's development process, but, commonly, this is an agonizing practice for teachers and students. Goals: to identify suggestions for improving evaluative practice in active learning in undergraduate health; Methods: qualitative study, carried out through semistructured individual interviews, with 25 professors from the Nursing and Medicine courses, in a public Higher Education Institution, in the central west region of the interior of São Paulo. The data were interpreted by the Content Analysis Technique, in the thematic modality, according to the stages Pre-analysis (organization of the data collected through successive readings), Exploration of the material (identification of the categories) and Treatment of the results: inference and interpretation (clarification of the meanings contained in the data collected and articulation between data interpretation, theoretical framework and study objectives). Results: identified three categories for improving evaluation in active health learning, being: Teacher development (pedagogical teacher training, especially in affective evaluation and better preparation to deal with relational, affective, communicational and group aspects), Adequacy of instruments and evaluation practices (standardization and implementation of evaluation instruments and adherence to more objective correction criteria) and Student orientation (means of training students about evaluation practices and their objectives, with emphasis on self-evaluation and peer evaluation). Final considerations: it is noted that the improvement of evaluative practice in active learning is inseparable from the training of teachers and students and the adoption of standardized assessment practices and instruments known to all its actors.

Keywords: Learning; Educational Measurement; Problem-Based Learning. 


\section{Introdução}

O momento avaliativo comumente é tido como angustiante, tanto para o avaliador quanto para 0 avaliado. Entretanto, esse processo não deveria ser considerado desta forma, especialmente quando se trata do uso de métodos de aprendizagem ativa (OliveiraBarreto et al., 2017).

É esperado que, na aprendizagem ativa, a avaliação seja uma ferramenta modulatória do processo de desenvolvimento do estudante, de modo que o docente aponte suas potências e fragilidades e o ajude a traçar um plano de aperfeiçoamento. Isso requer um docente habilidoso em invertigar, observar e acolher o estudante e, em contrapartida, um estudante capaz de receber críticas, se adaptar a mudanças e se empenhar com sua própria aprendizagem (Silva \& Gomes, 2018).

Além de possíveis fragilidades nesses aspectos, pesquisas revelaram outros pontos, também passíveis de melhoria, a fim de que a avaliação desempenhe seu desígnio. Dentre os quais, é relevante destacar a ênfase na participação oral do estudante, em detrimento da avaliação afetiva; a falta de uso de diferentes instrumentos de forma objetiva e padronizada e a realização da avaliação por diferentes docentes; o descompasso da combinação entre avaliação formativa e somativa bem como a necessidade de capacitação docente (Lemes et al., 2019; Lemes et al., 2020).

Assim, notou-se que inúmeros são os desafios que permeiam o sucesso dessa prática e, diante do exposto, emergiu a seguinte questão: "quais são as sugestões de aprimoramento da avaliação na formação em saúde desenvolvida por método de aprendizagem ativa?". Por tal razão, o presente estudo propôs-se a identificar sugestões de aprimoramento da prática avaliativa em aprendizagem ativa na graduação em saúde.

\section{Método}

A partir de uma abordagem qualitativa, com docentes da segunda série dos cursos de Enfermagem e Medicina, em uma Instituição de Ensino Superior (IES) pública que aderiu a aprendizagem ativa há 24 anos, o presente estudo foi desenvolvido.

A pesquisa qualitativa visa tornar a realidade, o mais fidedignamente possível, visível a olhos que encontram-se fisicamente distantes dela. Em outras palavras, por meio da apresentação de percepções, crenças, valores, caracterização, contexto sociocultural, busca torná-la evidente para que, então, seja possível a compreensão do fenômeno estudado e, enfim, sua interpretação (Minayo \& Costa, 2018). Visando a confiabilidade desse estudo e a minimização de aspectos subjetivos, seguiu-se os princípios do COREQ para sua realização (Tong et al., 2007).

Participaram do estudo 25 docentes, os quais foram selecionados por meio de sorteio, a partir de uma lista fornecida pelos coordenadores do curso e, na medida em que houve recusa ou dificuldades de marcar horário com o convidado, com pelo menos três tentativas, novo sorteio foi realizado. A amostra foi obtida por conveniência, por meio de contato pessoal com os docentes ao término de suas atividades educacionais ou via email e, quando se observou a saturação teórica, a coleta de dados foi encerrada (Minayo, 2017).

A IES preponente do estudo desenvolve seu processo de ensino por meio de métodos de aprendizagem ativa e o processo avaliativo é composto por exercício de avaliação cognitiva, quatro vezes ao ano, que tem como foco o conhecimento cognitivo em uma perspectiva interdisciplinar, envolvendo os aspectos biopsicossociais; autoavaliação e avaliação dos pares diariamente, ao fim das atividades; documento formal preenchido pelos docentes quatro vezes ao longo do ano letivo, contendo questões dissertativas acerca do desempenho individual do estudante em relação às habilidades, atitudes e conhecimentos cognitivos; portfólio reflexivo e atividade de simulação realizada individualmente pelos estudantes junto a atores que representam pacientes ao término da série. 
Visando avançar no processo de ensino e aprendizagem, os docentes participam semanalmente ou quinzenalmente de atividades de Educação Permanente (EP), propostas pelo Núcleo de Desenvolvimento Docente da IES, o que permite constante reflexão sobre a ação cotidiana e trocas de experiências entre os pares (Faculdade de Medicina de Marília, 2018; 2019).

Realizou-se a coleta de dados por meio de entrevistas semiestruturadas, de forma individual, que foram gravadas e transcritas na íntegra. A entrevista semiestruturada utiliza questões previamente formuladas e outras abertas, com o intuito de fornecer ao pesquisador um certo controle do que se deseja saber, ao passo que permite a reflexão espontânea do entrevistado acerca da temática. O uso do roteiro permite também maior uniformidade entre as entrevistas realizadas e ampara o pesquisador objetivamente em seus pressupostos de estudo (Minayo \& Costa, 2018).

As entrevistas foram realizadas no primeiro semestre de 2019, pela pesquisadora principal, em ambientes da instituição que garantiam o sigilo, após explicação do objetivo do estudo e procedimento da coleta de dados e agendamento prévio. Cada entrevista foi audiogravada e teve duração média de 20 minutos, sem necessidade de repetição. $O$ instrumento de coleta de dados abordou a caracterização dos participantes (sexo, faixa etária, estado civil, formação, titulação e tempo de atuação em método de aprendizagem ativa) e a questão norteadora "O que você poderia sugerir para melhoria do processo avaliativo?".

Os dados coletados foram interpretados à luz da técnica de análise de conteúdo na modalidade temática. Para tanto, percorreu-se suas três etapas, a saber, Pré-análise, Exploração do material e Tratamento dos resultados: inferência e interpretação. Durante a primeira etapa, o pesquisador pode organizar seu trabalho ao realizar "leituras flutuantes". A aproximação com os dados coletados a serem analisados permite uma visão geral dos mesmos e a percepção dos entrevistados sobre a temática e, ainda na préanálise, pode-se reformular hipóteses e objetivos de pesquisa, por meio da exaustividade, homogeneidade, pertinência e exclusividade, que orientarão interpretação e síntese posteriores (Bardin, 2011).

A segunda etapa, por sua vez, objetiva identificar o núcleo de compreensão do texto, a partir de temas que compreendam expressões significativas. Leitura da teoria, seguida dos dados, seguida de nova leitura da teoria consistem em um ciclo muitas vezes repetidos a fim de que ocorra a exploração adequada do material e se obtenha categorias coesas, as quais respeitem os seguintes aspectos: exclusão mútua, homogeneidade, pertinência, objetividade e fidelidade e produtividade (Bardin, 2011).

Finalmente, na terceira etapa organizar-se-á o tratamento dos resultados, por meio de inferência e interpretação. A inferência como técnica de tratamento de resultados é orientada por polos de comunicação (emissor, receptor, mensagem e canal) e ocupa-se em investigar causas a partir dos efeitos, a fim de esclarecer os significados contidos nos dados coletados. Quanto a interpretação dos dados, pressupõe a articulação entre interpretação dos dados, referencial teórico e objetivos do estudo (Bardin, 2011).

Esse estudo foi aprovado pelo Comitê de Ética em Pesquisa que envolve seres humanos da instituição proponente, sob o parecer número 2.975.092 e CAAE 91013218.6.0000.5413, e os participantes assinaram o Termo de Consentimento Livre e Esclarecido, conforme os pressupostos da Resolução no 510 (2016). Respeitando o sigilo, os participantes foram assim codificados: "D", em referência a "Docente", seguidos de sequência numérica crescente, ou seja, de "D1" à "D25".

\section{Resultados}

Dentre os 25 docentes que participaram desse estudo, $72 \%$ são do sexo feminino, $68 \%$ são casados e $60 \%$ tem idade entre 45 e 59 anos. Quanto à formação acadêmica, $52 \%$ dos participantes cursou Enfermagem e 52\% possui mestrado. Além disso, 84\% atua há mais de 10 anos em método de aprendizagem ativa. 
Vol. 7 | Investigação Qualitativa em Educação: Avanços e Desafios

A manifestação dos participantes em resposta à questão norteadora, indicou a seguinte categorização das sugestões de aprimoramento da avaliação em método de aprendizagem ativa: Desenvolvimento Docente; Adequação dos instrumentos e práticas avaliativas e Orientação aos estudantes.

\subsection{Desenvolvimento Docente}

Embora a IES ofereça encontros quinzenais de EP, os docentes manifestam seu desejo de maior investimento em sua formação.

"Esses momentos de EP tem que ser melhor investido mesmo para dar suporte para o docente, para dar segurança maior, pra gente discutir essas dificuldades." (D11)

"É importante fazer capacitações com os próprios professores, que às vezes a gente acha que está fazendo uma boa avaliação, mas nem sempre essa avaliação está sendo de forma adequada, ou se falta algum conteúdo pra gente estar avaliando esse aluno." (D24)

A maior fragilidade no tocante a capacitação avaliativa parece estar relacionada a avaliação afetiva, tanto em sua concepção, quanto na prática e no seguimento após identificar algum desvio no estudante.

"Um rigor maior nessa questão da avaliação emocional do indivíduo, [...] de ser mais cobrada, porque é uma questão muito íntima do indivíduo, que depois não se fala de diagnóstico, tem aquela questão da privacidade, mas acaba gerando essas dificuldades na sequência. Acho que precisa ser mais explicitado essa questão de o indivíduo estar bem emocionalmente para fazer um curso médico." (D16)

"Ter um fluxo de como encaminhar quando é problemas atitudinais, que às vezes eu acho que não é muito claro." (D23)

Outros aspectos apontados acerca da prática docente que também poderiam ser aprimorados, segundo os participantes são a capacidade de empatia, o feedback e a criação de oportunidades de aprendizado.

"Acho que os professores precisariam se sensibilizar mais na própria relação docente e estudante, para que essas avaliações possam vir de uma forma diferente." (D6)

"Outra coisa que eu acho que precisa melhorar é orientar as pessoas a deixar isso de uma forma bastante clara para o estudante, para ele reconhecer onde ele está "falho" para ele poder fazer a correção." (D23)

\subsection{Adequação dos Instrumentos e Práticas Avaliativas}

A padronização e a implementação de instrumentos avaliativos surgem como solução para tornar a avaliação mais objetiva e uniforme, beneficiando o estudante que a recebe e 0 docente que a faz.

"Os formatos de avaliação que escola têm são muito amplos, dando margem para diferentes avaliações do mesmo estudante. [...] Olhar para esses instrumentos seria um ponto importante: olhar a longitunalidade do currículo, os formatos de desempenho e discriminar os domínios que estão sendo considerados na avaliação." (D10) 
"Ter um instrumento de apoio para o tutor, onde ele consiga colocar, por exemplo, o aluno fez a leitura do caso, conseguiu ir a lousa, sabe, ter algo em que o tutor possa colocar um ' $X$ ' e colocar o número de vezes e ali ele vai então norteando. Ter um parâmetro e até auxiliaria para ele fazer sugestões no final. Então ter um instrumento a parte e que esse instrumento facilitasse o preenchimento mais fidedigno do formato." (D25)

Há também sugestões quanto a avaliação dissertativa, o Exercício de Avaliação Cognitiva (EAC):

"As questões do EAC se repetem muito em termo de conteúdo [...] acho que se mudasse seria bom, porque tira um pouco a estabilidade do aluno, porque ele ouve dos alunos dos outros anos. Ficando mais inseguro talvez se preocupassem um pouco mais ainda com a questão do método de estudo." (D13)

"Falando do EAC, se o professor fizesse uma pergunta tal que restringisse mais a resposta, de tal forma que o aluno respondesse ou isso ou aquilo, seria melhor." (D15)

\subsection{Orientação aos Estudantes}

Além da capacitação docente, os participantes apontaram a orientação do estudante como um fator que pode contribuir para o aprimoramento do processo avaliativo. Nesse contexto, insurgem a compreensão acerca da prática da autoavaliação e da avaliação dos pares.

"Acho que o nosso estudante também tem que entender sobre o processo de avaliação, para ele também poder estar ciente de quais estratégias de avaliação nós utilizamos, o porquê nós utilizamos, para que ele também valorize e possa ser sincero quando faz uma autoavaliação, quando ele avalia o seu colega, quando ele avalia o professor, e, reforçando, a capacitação docente." (D3)

"O que eu percebo é que muitas vezes eles acabam não criticando um ao outro por até coleguismo. Eu acho que tem que enfatizar mais que a avaliação é para o bem do próprio aluno. Quer dizer, ele criticar um aluno de forma cordial, respeitosa, acaba ajudando um outro colega que não esteja estudando direito, saber receber a crítica. [...] Para os alunos, deixar claro a importância da crítica construtiva, da avaliação, de um criticar o outro." (D19)

\section{Discussão}

Em virtude do que foi exposto pelos participantes, percebeu-se o interesse por parte dos mesmos em novos investimentos da IES em questão, visando melhor qualificação docente para atuação nos cenários educacionais. Como qualquer profissão, a docência precisa ser edificada. De acordo com Souza e Passalacqua (2019), é necessário o momento contextualizado, formal, fundamentado e sistematizado para fornecer subsídios a esses profissionais. Portanto, essa edificação requer mais que leituras de textos e atividades teóricas, solicitando também atividades práticas e estágios supervisionados.

Todavia, a prática docente no ensino superior possui caráter amador e diletante, na figura de profissionais-professores, oposto ao que ocorre nos níveis básicos, onde há professores-profissionais, com formação sólida em licenciatura no tema lecionado (Quintanilha et al., 2020). Desse modo, é, em sua maioria, alicerçada nas vivências prévias, ainda como estudante ou já no exercício da profissão (Zimmermann et al, 2016).

Pesquisa realizada com cento e oitenta docentes do curso de Medicina (Quintanilha et al., 2020) apontou que a maioria não considerava sua carreira docente como principal. 
Tal fato, embora não abordado na presente pesquisa, parece contribuir para o empenho individual em sua própria formação como docente, pois embora os participantes dessa pesquisa afirmassem que cursos de capacitação pedagógica pudessem colaborar com sua performance, uma parcela significativa desses alegaram que não os realizavam quando fornecidos pela IES.

Desse modo, ressalta-se a importância da união entre organização de cursos didáticopedagógicos por parte das instituições de ensino e a adesão por parte dos docentes. Como relatado por inúmeros participantes dessa investigação, a IES fornece quinzenalmente encontros de EP, o que, ainda de acordo com os relatos, não tem sido suficiente para sanar lacunas e promover segurança na atuação desses profissionais. Assim, levante-se a questão da volição e disponibilidade desses profissionais em participar desses encontros e torná-los mais eficazes. Por outro lado, é relevante destacar também que, diante da sinalização da não eficácia dos encontros de EP, a IES pode lançar mão de novas estratégias para promover maior adesão docente.

Para Zimmermann et al. (2016), a dimensão coletiva de formação docente possibilita um espaço de reflexão, aprendizado e é potente para transformações no modo de atuar nas resoluções de problemas cotidianos. Nesse espaço, o conteúdo deve ser de interesse do docente, com carga horária adequada e promover participação ativa, junto a atividades que permitam relação teórico-prática.

Outro aspecto apontado por parte dos entrevistados foi a avaliação afetiva. Segundo os mesmos, essa ferramenta possui fragilidades e carece de ajustes para que sua aplicação seja eficiente. Neste sentido, além da capacitação docente, aderir o uso de escalas validadas pode tornar a avaliação afetiva mais confiável e valorizada, ao passo que permitem a identificação de alterações afetivas e atitudinais a serem trabalhadas. Sabese que valores como compromisso com excelência, interesse por conhecimento, empatia, honestidade, altruísmo, respeito pelo paciente e pelos pares, capacidade de reflexão raramente são abordados durante a graduação, ainda que fundamentais para consolidar uma prática ética, por isso a implementação de ferramentas que abordem tais valores torna-se importante para o desenvolvimento do estudante e sua formação como um profissional de excelência (Carneiro et al., 2020).

Identificado algum transtorno mental nos estudantes, há comumente empasse: a comunicação deficiente entre serviços de apoio para prevenção de transtornos mentais e estudantes, previamente descrito por Brown (2016). Desse modo, é preciso que as IES tomem partido e invistam em serviços e programas com vistas a aconselhar, acompanhar, encaminhar e orientar estudantes de acordo com suas necessidades. Tal amparo é significativamente importante, pois o equilíbrio de aspectos psicológicos é, junto a aspectos sociais, um fator mantenedor do engajamento estudantil e impacta diretamente seu desempenho acadêmico. O contrário também é verdadeiro: a desarmonia psicológica ou social minimiza a qualidade das atividades dos estudantes, o que reforça a importância de o estudante ter acesso a uma rede de apoio e ser consciente dela (Silva et al., 2018).

No tocante à figura docente, Martins et al. (2019) apontam que a eficácia de sua performance em aprendizagem ativa tem relação com o domínio do conteúdo e a competência em facilitação de grupos. Com o domínio do conteúdo, o docente pode ajustar a compreensão do estudante, ao passo que por meio de acolhimento, comunicação informal e empatia o docente realizará congruência social, também fundamental para o desenvolvimento do estudante. Pode-se notar então que, além de desenvolver habilidades pedagógicas, dominar o método de ensino, aprendizagem e avaliação e o uso de instrumentos e escalas e o conteúdo programático, cabe ao docente desenvolver habilidades atitudinais, especialmente as que avaliará em seus estudantes.

Ainda nessa vertente, de acordo com Lucey et al. (2020), o ambiente em que as estratégias de avaliação são implementadas também é apontado como importante, já que afeta o clima vivenciado pelos estudantes e, portanto, a oportunidade que esses têm de desempenhar suas atividades e serem avaliados em suas habilidades máximas. 
Ademais, o modo com que os resultados dos processos de avaliação são compartilhados deve criar oportunidades equitativas para todos os estudantes, independentemente de sua classe social ou características pessoais. Portanto, o programa de avaliação deve articular explicitamente critérios de avaliação à igualdade de oportunidades de aprendizagem e feedback formativo, para diminuir o impacto deletério do viés inconsciente dos avaliadores nas notas dos estudantes. Pesquisa conduzida por Montes et al. (2019) descreveu algumas características essenciais ao feedback, entre elas, ser assertivo, descritivo, específico, oportuno e respeitoso. Além disso, que seja iniciado por aspectos positivos, seguidos de negativos. Outro ponto destacado foi que se evite elencar muitas fragilidades em uma mesma devolutiva.

Quanto a adequação dos instrumentos e práticas avaliativas, a crítica dos entrevistados apontou a necessidade de padronização e objetividade na avaliação, de tal modo que sirvam para nortear a aprendizagem dos estudantes e otimizar o trabalho docente. A fim de propiciar essas condições, alguns aspectos são indispensáveis a quaisquer instrumentos e práticas, como validade, reprodutibilidade, equivalência, viabilidade, efeito educacional, efeito catalítico e aceitabilidade. Em outras palavras, para que um mesmo aluno seja avaliado por diferentes docentes e essas avaliações sejam o mais coerente possível entre si, é preciso que o programa de avaliação e as ferramentas sejam desenhados com essa finalidade e que sua aplicação seja também padronizada. Ainda sobre o desenho desse processo, ao aplicar uma avaliação deve-se ter em mente o resultado esperado, a partir do que se pretende avaliar, o qual não deve superar ou subestimar o desempenho subsequente (Lucey et al., 2020). Preston et al. (2019) defendem que a adoção de critérios de avaliação explícitos, construídos conjuntamente e conhecidos por docentes e estudantes, tendem a melhorar o desempenho acadêmico e a satisfação com a prática avaliativa. Tornar a avaliação mais criteriosa, objetiva e produtiva, contribui tanto para a devolutiva docente quanto para a percepção do estudante sobre seu desempenho.

Ainda sobre a adequação dos instrumentos e práticas avaliativas, os entrevistados destacaram a avaliação dissertativa, sugerindo especialmente a adequação de sua correção. Embora seja respondido de modo livre, a correção do EAC também necessita pautar-se em critérios objetivos e previamente estabelecidos, fornecendo aos estudantes o mesmo crivo e as mesmas oportunidades de aprendizagem. A aplicação desse tipo de instrumento visa investigar habilidades de argumentação, criatividade, memorização, síntese, entre outras, além de permitir a correlação entre saberes (Vito \& Szezerbatz, 2017).

Finalmente, parte dos entrevistados relatou a necessidade de orientação aos estudantes. Sobre esse ator, características pessoais vistas como limitantes para seu desenvolvimento e avaliação podem ser melhoradas no processo de aprendizagem ativa. Isso porque esse modelo é estruturado de forma a oferecer ao estudante situações nas quais ele deve agir com autonomia e postura profissional, solucionar problemas, relacionar-se com outros estudantes, docentes e pacientes bem como identificar pontos positivos e negativos, como nos momentos de avaliação e autoavaliação. Assim, ao passo que estudantes forem mais bem conscientizados a respeito dos objetivos educacionais, das práticas avaliativas e da função da avaliação no processo de ensino e aprendizagem, em seu desenvolvimento, obterá melhores resultados a partir dessas (Oliveira-Barreto et al., 2017).

Sobre a avaliação dos pares e a autoavaliação, Roberts et al. (2017) elencaram como aspectos positivos dessa prática sua contribuição com a dinâmica do grupo, amadurecimento das relações interpessoais, habilidade de fazer e receber críticas e adoção de postura colaborativa. Além disso, tais práticas auxiliam na percepção mais verídica de cada estudante por parte dos docentes. 


\section{Considerações finais}

Nesta pesquisa, a abordagem qualitativa foi fundamental para identificar sugestões de aprimoramento da prática avaliativa em aprendizagem ativa na graduação em saúde, pois permitiu que tanto manifestações objetivas quanto subjetivas fossem reunidas, compreendidas e interpretadas. Assim, permite ao leitor aproximar-se dessa realidade e, da mesma forma, compreendê-la e interpretá-la.

Em síntese, docentes entendem a necessidade de capacitação contínua para melhor manejo das práticas avaliativas, porém, há baixa adesão nos programas ofertados pela IES em que foi realizada esta pesquisa.

Além disso, os entrevistados apontaram para a necessidade de reestruturação das práticas avaliativas, considerando o aluno como cerne desse momento. Fato este evidenciado pela literatura científica como de extrema relevância no processo de ensino e de aprendizagem, que coloca em mister o uso adequado do feedback e de critérios objetivos para avaliar o estudante.

Evidenciou-se também a necessidade de conscientização e participação ativa do estudante nesse processo, por meio de avaliação por pares e auto-avaliação, para aprimoramento de atitudes, conhecimentos e habilidades.

Vale ressaltar que, além do empenho de docentes e estudantes, tal aprimoramento requer o compromisso institucional de promover momentos de conscientização e oferta de espaços reflexivos, acerca da prática avaliativa, seus objetivos, instrumentos, formas de aplicação e critérios.

Por esse caminho, entende-se que a prática avaliativa pode ser não apenas aprimorada, mas transformada em prática acolhedora e desejada por avaliadores e avaliados.

Como limitação da pesquisa, há o fato de ter sido realizada em apenas uma IES.

\section{Referências}

Bardin, L. (2011). Análise de Conteúdo (5ª ed). Lisboa: Edições 70.

Brown, P. (2016). The invisible problem? Improving students' mental health. Oxford: Higher Education Policy Institute Report 88.

Carneiro, M. A., Cunha, S. M., Feitosa, E. S., Sá, R. B., \& Brilhante, A. V. M. (2020). O profissionalismo e suas formas de avaliação em estudantes de Medicina: uma revisão integrativa. Interface - Comunicação, Saúde, Educação, 24, e190126. doi: 10.1590/interface.190126

Faculdade de Medicina de Marília. (2018). Caderno de avaliação: cursos de Medicina e Enfermagem. Marília: Faculdade de Medicina de Marília.

Faculdade de Medicina de Marília. (2019). Necessidades de Saúde 2 e Prática Profissional 2- 2a série dos cursos de Medicina e Enfermagem. Marília: Faculdade de Medicina de Marília.

Lemes, M. A., Marin, M. J. S., Lazarini, C. A., Bocchi, S. C. M., Ghezzi, J. F. S. A., \& Higa, E. F. R. (2020). Avaliação em aprendizagem ativa: representações sociais dos estudantes de enfermagem. New Trends in Qualitative Research, 2, 549-563. doi: 10.36367/ntqr.2.2020.549-563

Lemes, M. A., Marin, M. J. S., Lazarini, C. A., Peres, C. R. F. B., \& Higa, E. F. R. (2019). Avaliação em metodologias de aprendizagem ativa: percepção docente. In: Costa, A. P., Sá, S. O., Castro, P., \& Souza, D. N. (Eds.), Atas do 8ํㅡㄹ Congresso Ibero-Americano em Investigação Qualitativa (pp. 458-467). Recuperado de https://proceedings.ciaiq.org/index.php/CIAIQ2019/article/view/2184/2109

Lucey, C. R., Hauer, K. E., Boatright, D., \& Fernandez, A. (2020). Medical Education's Wicked Problem: Achieving Equity in Assessment for Medical Learners. Academic Medicine, 95, S98-S108. doi: 10.1097/ACM.0000000000003717 
Martins, A. C., Falbo Neto, G., \& Silva, F. A. M. (2019). Características do Tutor Efetivo em ABP Uma Revisão de Literatura. Revista Brasileira de Educação Médica, 42(1), 105-114.

Minayo, M. C. S. (2017). Amostragem e saturação em pesquisa qualitativa: consensos e controvérsias. Revista Pesquisa Qualitativa, 5(7),1-12. Recuperado de https://editora.sepq.org.br/index.php/rpq/article/view/82

Minayo, M. C. S., \& Costa, A. P. (2018). Fundamentos teóricos das técnicas de investigação qualitativa. Revista Lusófona de Educação, 40(40), 139-153. Recuperado de https://revistas.ulusofona.pt/index.php/rleducacao/article/view/6439

Montes, L. G., Rodrigues, C. I. S., \& Azevedo, G. R. (2019). Avaliação do processo de feedback para o ensino da prática de enfermagem. Revista Brasileira de Enfermagem, 72(3), 663-670. doi: 10.1590/0034-7167-2018-0539

Oliveira-Barreto, A. C., Guedes-Granzotti, R. B., Domenis, D. R., Pellicani, A. D., Silva, K., Dornelas, R., \& César, C. P. H. A. R. (2017). Métodos de avaliação discente em um curso de graduação baseado em metodologias ativas. Revista Ibero-Americana de Estudos em Educação, 12(02), 1005-1019. doi: /10.21723/riaee.v12.n2.8745

Quintanilha, L. F., Farias, C. S. S., \& Andrade, B. B. (2020). Formação e envolvimento pedagógico entre docentes do ensino superior em saúde: uma análise dos cursos médicos. Revista Internacional de Educação Superior, 7, e021026. doi: 10.20396/riesup.v7i0.8659850

Resolução n. 510, de 7 de abril de 2016. (2016). Sobre a Ética na Pesquisa na área de Ciências Humanas e Sociais. Diário Oficial da União, Brasília, DF.

Roberts, C., Jorm, C., Gentilcore, S., \& Crossley, J. (2017). Peer assessment of professional behaviours in problem-based learning groups. Medical Education, 51(4), 390-400. doi: 10.1111/medu.13151

Silva, A. L., \& Gomes, A. M. (2018). Avaliação educacional: concepções e embates teóricos. Estudos em Avaliação Educacional, 29(71), 350-385. doi: 10.18222/eae.v29i71.5048

Silva, J. O. M., Pereira Junior, G. A., Coelho, I. C. M. M., Picharski, G. L., \& Zagonel, I. P. S. (2018). Engajamento entre Estudantes do Ensino Superior nas Ciências da Saúde (Validação do Questionário Ultrecht Work Engagement Scale (UWES-S) com Estudantes do Ensino Superior nas Ciências da Saúde). Revista Brasileira de Educação Médica, 42(2), 1525. doi: 10.1590/1981-52712015v42n2rb20170112

Souza, N. C. A. T., \& Passalacqua, F. G. M. (2019). O processo de construção da profissionalidade docente: aspectos concernentes à formação inicial. Revista Internacional De Educação Superior, 5, e019018. doi: 10.20396/riesup.v5i0.8653296

Tong, A., Sainsbury, P., \& Craig, J. (2007). Critérios consolidados para relatar pesquisas qualitativas (COREQ): uma lista de verificação de 32 itens para entrevistas e grupos de foco. International journal for quality in health care, 19(6), 349-357. doi:10.1093/intqhc/mzm042

Vito, D. Z., \& Szezerbatz, R. P. (2017). A avaliação no ensino superior: a importância da diversificação dos instrumentos no processo avaliativo. Educere - Revista da Educação da UNIPAR, 17(2), 221-236. doi: 10.25110/educere.v17i2.2017.6598.

Zimmermann, M. H., Silveira, R. M. C. F., \& Gomes, R. Z. (2019). O Professor e a arte de avaliar no ensino médico de uma universidade no Brasil. Revista Brasileira de Educação Médica, 43(3), 5-15. doi: 10.1590/1981-52712015v43n3rb20180167 